\title{
Secondary renal tubular dysgenesis in a newborn exposed to angiotensin II receptor antagonist during gestation
}

\section{Clinical course of the patient}

A male infant weighing 2,150 g was delivered. The Apgar scores were 7 at 1 minute and 9 at 5 minutes. At birth, he was meconium-stained. Birth weight and height of the infant were below the 10th percentile for its gestational age. His mother, a 38-year-old woman, was referred to our center at 36 weeks of gestation for intrauterine growth restriction and oligohydramnios detected at 30 weeks. It was her third pregnancy followed by 2 previous abortions ( 1 spontaneous abortion, 1 artificial abortion) that occurred 5 to 10 years ago. Reduced fetal movements had been noted in the preceding 4 weeks. Nothing relevant could be found other than oligohydramnios from antenatal ultrasound. She took a tablet of Sevikar (Daiichi Sankyo Europe $\mathrm{GmbH}$, Munich, Germany) containing $40 \mathrm{mg}$ of olmesartan medoxomil and $5 \mathrm{mg}$ of amlodipine (as amlodipine besilate) once a day because of hypertension diagnosed 2 years before pregnancy and had smoked half a pack of cigarettes a day throughout pregnancy. She denied any family history of genetic diseases.

The patient presented with respiratory failure and bilateral pneumothorax although there were no features of typical Potter sequence. He presented with rapid respiration and subcostal retraction on admission. His respiratory status continued to deteriorate and he received mechanical ventilation after surfactant administration. During the 1st day of life, he developed severe arterial hypotension (mean arterial blood pressure: 10-20 $\mathrm{mmHg}$ ) which poorly responded to fluid boluses, catecholamine therapy (dopamine, dobutamine, noradrenaline, and epinephrine), and hydrocortisone therapy. On the 2nd hospital day, persistent pulmonary hypertension of the newborn (PPHN) was confirmed by Doppler echocardiography showing rightto-left shuntings through a widely patent ductus arteriosus and a patent foramen ovale and intravenous milrinone and inhaled nitric oxide were administered. He was anuric since birth, which persisted without response to fluid restriction after attempting fluid resuscitation or diuretic therapy. Renal ultrasonography demonstrated diffuse increased cortical echogenicity of the right and left kidneys and increased resistive index value of the left kidney (Fig. 1A-C). On the 5th day of life, acute peritoneal dialysis (PD) was started for persistent anuria and renal failure (serum creatinine, $3.76 \mathrm{mg} / \mathrm{dL}$; blood urea nitrogen, $36.5 \mathrm{mg}$ /

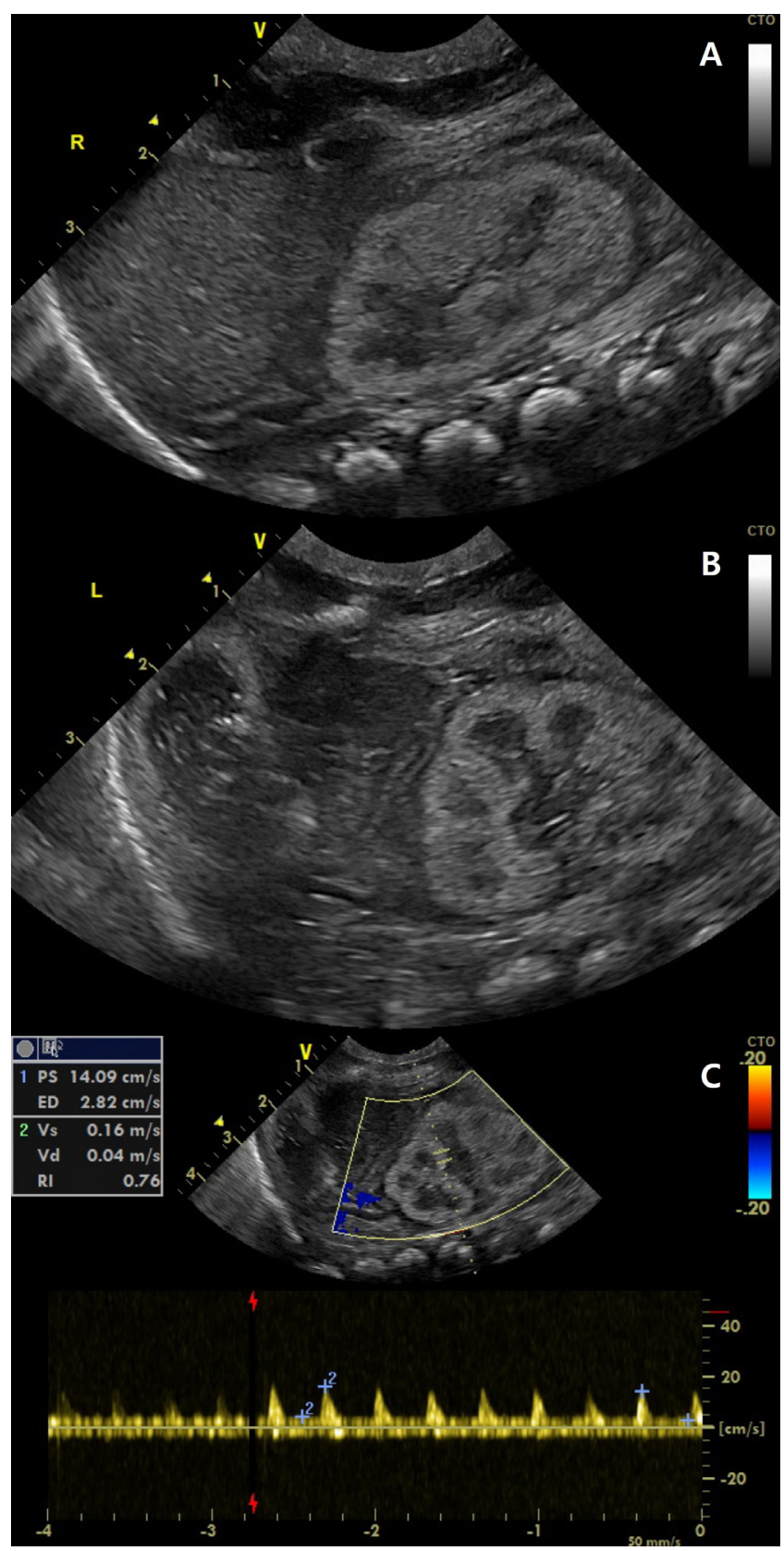

Fig. 1. Renal ultrasonogram showing diffuse increased cortical echogenicity of the right (A) and left kidneys (B) and increased resistive index value (C) of the left kidney. The right and left kidneys were $3.1 \mathrm{~cm}$ long each. 
dL). Both hypotension and PPHN were alleviated after PD. On the 7th day of life, because of blood in the dialysis fluid drainage and free air on abdomen x-ray, we made an exploratory laparotomy confirming multiple perforations of the small intestine and transverse colon, and converted PD to ccontinuous renal replacement therapy (CRRT). The kidney biopsy performed during surgery confirmed the absence or underdevelopment of proximal tubules with the intervening tubules small and undifferentiated, compatible with renal tubular dysgenesis (RTD) (Fig. 2A, B). His karyotype was 46, XY and no ACE mutation was detected by conventional Sanger sequencing. CRRT was interrupted and resumed repeatedly due to uncontrolled bleeding caused by severe disseminated intravascular coagulation. On the 12th hospital day, CRRT was stopped due to active bleeding of surgical site and unfortunately, the parents did not agree to resume CRRT after that. On the 25th hospital day, he died of cardiac arrest due to hyperkalemia.

Q: Which of the following drugs is not known to adversely affect fetal kidney development?

(1) Racetam anticonvulsant

(2) Prostaglandin synthetase inhibitors

(3) Angiotensin-converting enzyme inhibitor

(4) Angiotensin II receptor blocker

\section{Discussion of the disease}

RTD is a congenital renal abnormality characterized by absence or poor development of proximal tubules and/or immature glomeruli resulting in reduced fetal urine production, oligohydramnios, the Potter sequence, and skull ossification defects. ${ }^{1,2)}$ Affected individuals usually die before birth or die soon after birth from anuria, respiratory failure, or refractory hypotension. Rarely, with treatment, they survive into childhood.

While mutations in the ACE, AGT, AGTR1, or REN gene in- volved in the renin-angiotensin system (RAS) can cause RTD, secondary RTD acquired after in-utero exposure to various drugs have been reported. ${ }^{3)}$ Renal failure in the neonate associated with poor tubular differentiation similar to inherited RTD could be induced by maternal use of RAS blockers, indomethacin, and other prostaglandin synthetase inhibitors. ${ }^{4)}$

There are several hypotheses for the mechanism by which angiotensin-converting enzyme inhibitors and angiotensin II receptor blockers (ARBs) cause fetal renal toxicity. ${ }^{5)}$ Considering that RTD could also be caused by severe cardiomyopathies or renal artery stenosis, the most likely mechanism leading to RTD is that these drugs block the RAS and thus decrease perfusion of the fetal kidney during nephrogenesis. ${ }^{6}$ RAS blockers are known to affect fetal kidneys in the second and third trimesters of pregnancy. ${ }^{7}$ It was reported that even though the mother took the drug in the second or third trimester of pregnancy, the amniotic fluid volume was normalized after the drug was discontinued before 34 to 36 weeks of gestation when nephrogenesis is terminated and the baby did not show renal impairment.7) Based on this report, if the mother stops taking RAS blockers before 34 to 36 weeks of pregnancy, when kidney formation is complete, the amount of amniotic fluid can be expected to normalize thereafter. Our patient did not have the opportunity to achieve normal fetal renal development because he was exposed to ARBs throughout pregnancy. Two Korean newborn cases related to RAS blockers have been reported but they showed reversible renal dysfunction and a kidney biopsy was not considered. ${ }^{8,9)}$

Physicians should educate pregnant women to discontinue the use of RAS blockers when pregnancy is confirmed. We should consider any degree of RAS fetopathy in a newborn born to a hypertensive mother who had use of any RAS blockers during her pregnancy.

\section{Answer: (1) Racetam anticonvulsant}

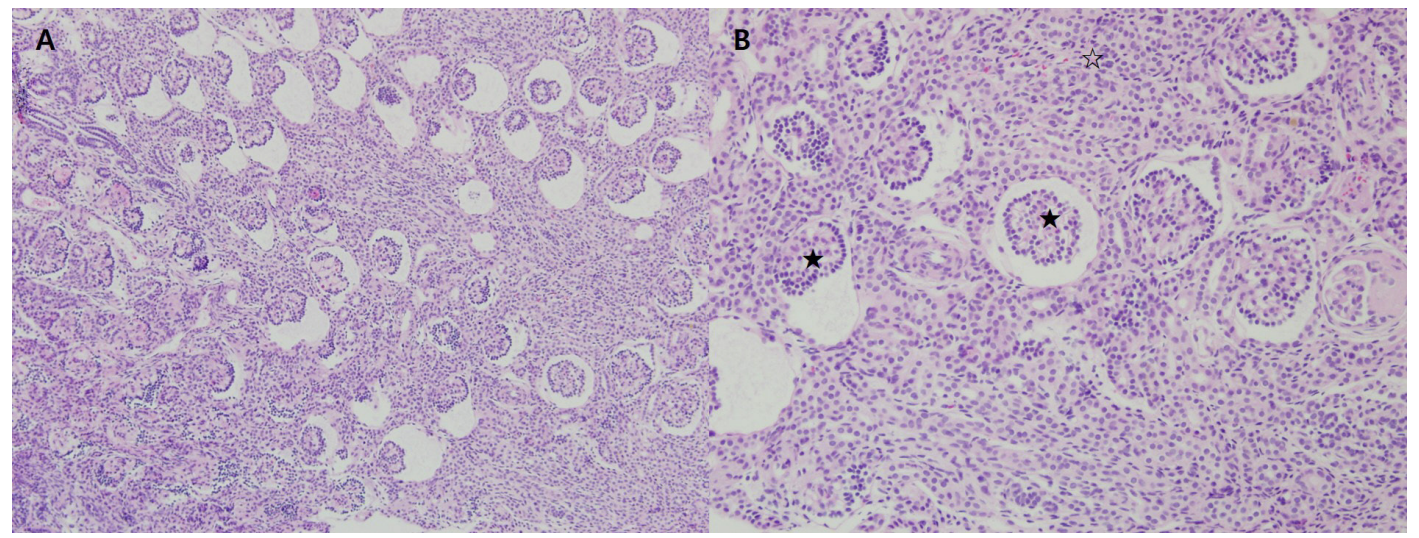

Fig. 2. Histopathology of the biopsied kidney tissue. (A) Narrow gaps between the glomeruli and an increased number of glomeruli are shown (hematoxylin and eosin staining, $\times 100$ ). (B) The glomeruli are small and immature, showing dilated Bowman capsules and no capillary openings $(\star)$. The intervening tubules are small and appear undifferentiated. No proximal tubules could be identified. All cortical tubules resemble distal tubules and are lined with immature primitive cells without

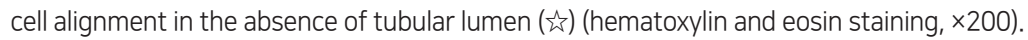




\section{Key message}

Question: The use of renin-angiotensin system (RAS) blockers during pregnancy causes lethal neonatal complications.

Finding: A neonate, whose mother took a RAS blocker during pregnancy, was diagnosed with renal tubular dysgenesis. He presented with respiratory failure, severe refractory hypotension, and anuria requiring continuous dialysis.

Meaning: Physicians should consider any degree of RAS fetopathy in a newborn born to a hypertensive mother who had use of RAS blockers during her pregnancy.

\section{Conflicts of interest}

No potential conflict of interest relevant to this article was reported.

Mi Hyeon Gang, MD, Yong Wook Lee, MD, Mea-young Chang, $\mathrm{MD}, \mathrm{PhD}$

Department of Pediatrics, Chungnam National University Hospital, Chungnam National University College of Medicine, Daejeon, Korea

Corresponding author: Mea-young Chang, $\mathrm{MD}, \mathrm{PhD}$

Department of Pediatrics, Chungnam National University Hospital, Chungnam National University College of Medicine, 282, Munhwa-ro, Jung-gu, Daejeon 35015, Korea

\E-mail: mychang@cnuh.co.kr

https://orcid.org/0000-0001-5368-3296

See the commentary "Inhibition of the renin-angiotensin system during fetal kidney development" via https:/doi.org/ 10.3345/cep.2020.01228.

\section{References}

1. Bullo M, Tschumi S, Bucher BS, Bianchetti MG, Simonetti GD. Pregnancy outcome following exposure to angiotensin-converting enzyme inhibitors or angiotensin receptor antagonists: a systematic review. Hypertension 2012;60:444-50.

2. Prasad N, Gulati S, Jain M, Tewari P. An unusual case of neonatal anuria. Indian Pediatr 2003;40:258-60.

3. Uematsu M, Sakamoto O, Ohura T, Shimizu N, Satomura K, Tsuchiya S. A further case of renal tubular dysgenesis surviving the neonatal period. Eur J Pediatr 2009;168:207-9.

4. Koklu E, Gurgoze M, Akgun H, Ozturk MA, Poyrazoglu MH. Renal tubular dysgenesis with atypical histology and in-utero exposure to naproxen sodium. Ann Trop Paediatr 2006;26:241-5.

5. Vendemmia M, Garcia-Méric P, Rizzotti A, Boubred F, Lacroze V, Liprandi A, et al. Fetal and neonatal consequences of antenatal exposure to type 1 angiotensin II receptor-antagonists. J Matern Fetal Neonatal Med 2005;18:137-40.

6. Gubler MC, Antignac C. Renin-angiotensin system in kidney develop ment: renal tubular dysgenesis. Kidney Int 2010;77:400-6.

7. Spaggiari E, Heidet L, Grange G, Guimiot F, Dreux S, Delezoide AL, et al. Prognosis and outcome of pregnancies exposed to renin-angiotensin system blockers. Prenat Diagn 2012;32:1071-6.

8. Ra JJ, Eun HS, Lee SM, Park MS, Namgung R, Lee C, et al. A case of neonate with acute renal failure after maternal treatment with angiotensin II receptor blocker. Korean J Perinatol 2012;23:286-91.

9. Ri S, Shin J, Je BK, Choi BM. Hypocalvaria of newborn infant: intrauterine exposure to an angiotensin receptor blocker. Perinatology 2017; 28:103-7.

How to cite this article: Gang MH, Lee YW, Chang MY. Secondary renal tubular dysgenesis in a newborn exposed to angiotensin II receptor antagonist during gestation. Clin Exp Pediatr 2021;64:136-8. https://doi.org/10.3345/cep.2020.00752 